sequelae. Gangrenous cholecystitis or appendicitis is reported in less than a dozen patients. ${ }^{4}$

In the present case, the clinical presentation reflected occlusion of meso-appendicular artery branches causing distal ischaemia of the appendix. The normal white cell count was surprising in a patient who proved to have pelvic sepsis and fever and it is possible that chronic autoimmune disease depressed his systemic inflammatory response. There was no hypertension. Antiphospholipid syndrome is itself associated with vasculitis, but antiphospholipid antibodies are seldom detected in polyarteritis nodosa. ${ }^{5}$ Prompt histological reporting spared this patient reoperation and led to effective long-term management.

\title{
REFERENCES
}

1 Jennette JC, Falk RJ. Update on the pathology of vasculitis. In: Schoen FJ, Gimbrone MA, eds. Cardiovascular Pathology: Clinico-pathologic Correlations and Pathogenic Mechanisms. Baltimore: Williams \& Wilkins, 1995

2 Guillevin L, Du LTH, Godeau P, Jais P, Wechsler B. Clinical findings and prognosis of polyarteritis nodosa and Churg-Strauss angiitis: a study in 165 patients. Br J Rheumatol 1998;27:258-64

3 Matolo NM, Albo N. Gastrointestinal complications of collagen vascular diseases: surgical implications. Am J Surg 1971;122:678-82

4 Colton CL, Butler TJ. The surgical problem of polyarteritis nodosa. Br J Surg 1967;54:393-6

5 Norden DK, Ostrov BE, Shafritz AB, Von Feldt JM. Vasculitis associated with antiphospholipid syndrome. Semin Arthritis Rheum 1995;24:273-81

\section{Uveitis in a patient receiving rifabutin for Crohn's disease}

\section{O Awotesu ${ }^{1} \quad T$ Missotten MD² \\ M C Pitcher LMD FRCP 3 W A Lynn MD FRCP 4 \\ S Lightman PhD FRCOphth ${ }^{5}$}

J R Soc Med 2004;97:440-441

Some clinicians are now treating Crohn's disease with rifabutin and a macrolide antibiotic ${ }^{1,2}$ because of evidence that Mycobacterium paratuberculosis contributes to the pathogenesis. In these circumstances, the development of uveitis may or may not be a rifabutin side-effect.

${ }^{1}$ Barts \& The London School of Medicine \& Dentistry, London E1; ${ }^{2}$ Moorfields Eye Hospital, London EC1; ${ }^{3}$ Northwick Park Hospital, Harrow; ${ }^{4}$ Infection \& Immunity Unit, Ealing Hospital, Southall; ${ }^{5}$ Institute of Ophthalmology, Moorfields Eye Hospital, City Road, London EC1V 2PD, UK

Correspondence to: Professor S Lightman

E-mail: s.lightman@ucl.ac.uk

\section{CASE HISTORY}

A Somali woman aged 22 with Crohn's disease developed redness, ache and a hypopyon first in the left eye then in the right eye, three weeks after starting treatment with rifabutin $300 \mathrm{mg}$ daily and clarithromycin $250 \mathrm{mg}$ twice daily. Her Crohn's disease had been diagnosed 10 years previously and treated for some years in the Middle East. On arrival in the UK at age 18 she was experiencing diarrhoea from severe colonic ulceration with painful perineal involvement and rectovaginal fistulating disease. The fistulae were laid open and seton drainage was established. For the next 2 years she took azathioprine $2 \mathrm{mg} / \mathrm{kg}$ but the disease did not respond. A defunctioning loop ileostomy was then fashioned and she came to proctocolectomy the following year. Subsequently there was global clinical improvement but she was troubled by an offensive discharge from a non-healing perineal wound cavity, communicating with the posterior wall of the vagina, for which she needed to wear a pad. MRI scanning of the pelvis confirmed inflammation within the proctectomy bed extending into the presacral space. At the time rifabutin and clarithromycin were started, she had never experienced ocular symptoms.

On ophthalmic examination she had bilateral anterior uveitis but good vision. Topical steroids were prescribed and the systemic medication was stopped, whereupon the symptoms resolved completely. About a month later, two days after restarting rifabutin and clarithromycin at the same dose as before, she reported sudden onset of pain and blurred vision in the left eye. On examination her visual acuity was $6 / 5$ right eye and $6 / 18$ left eye. A hypopyon was obvious in the left eye (Figure 1) and slit lamp examination showed numerous cells in the anterior chamber as well as in the vitreous. No retinal lesion was seen. Again the rifabutin and clarithromycin were stopped and the uveitis settled with topical steroids.

\section{COMMENT}

Patients with Crohn's disease can get uveitis as part of their disease process but hypopyon is unusual in these circumstances. Infection in any organ can also spread to the eye via the bloodstream and produce inflammation, often with a hypopyon, which takes many weeks to settle. In this patient, however, there are good reasons for thinking that the uveitis was drug-associated - namely, the onset soon after initiation of rifabutin and clarithromycin therapy, the rapid resolution when the treatment was stopped and the prompt recurrence when it was resumed.

Rifabutin-associated uveitis, which may be either unilateral or bilateral, typically presents as an anterior uveitis with a hypopyon. It can develop as early as two weeks and as late as nine months after the start of therapy and usually resolves within days when the drug is stopped 


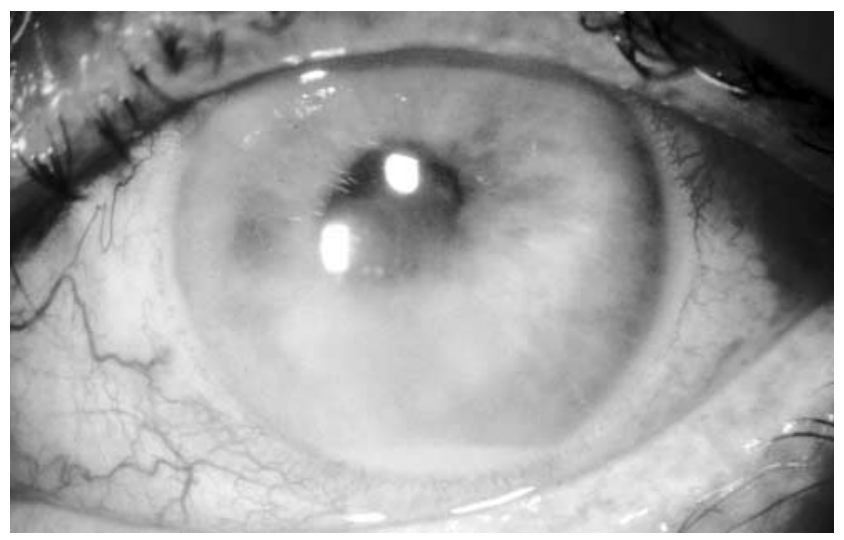

Figure 1 Hypopyon in patient with uveitis taking rifabutin and clarithromycin (left eye)

and the eye is treated with topical steroids. ${ }^{3,4}$ The condition is well reported in patients whose $M$. avium complex infection is HIV-associated, but immunocompetent individuals have also been affected. ${ }^{5}$ There seems to be a dose effect, with higher incidence and earlier onset with $600 \mathrm{mg}$ than with $300 \mathrm{mg}$ - cumulative risk at seven months $48 \%$ and $13 \%$. With rifabutin withdrawal, uveitis lasted 4 days; with rifabutin continuation it persisted for nearly seven weeks. Low body weight increased the risk. ${ }^{6}$

The mechanism of rifabutin-associated uveitis is unknown. Some workers have proposed a JarischHerxheimer-like reaction due to release of toxins by lysed bacteria. Against this hypothesis is the fact that, in M. avium infection, uveitis develops long after sterilization has been achieved whereas Herxheimer reactions occur soon after the start of treatment. Also, uveitis is not a side-effect of other drugs (such as clarithromycin) active against this infection.

In the two published studies on Crohn's disease treated with rifabutin, ${ }^{1,2}$ the incidence of uveitis was $4 / 46$ and $1 / 7$. Gastroenterologists and others using rifabutin for this purpose need to be aware of this side-effect and warn the patient.

\section{REFERENCES}

1 Shafran I, Kugler L, El-Zaatari FA, Naser SA, Sandoval J. Open clinical trial of rifabutin and clarithromycin therapy in Crohn's disease. Dig Liver Dis $2002 ; 34: 22-8$

2 Gui GP, Thomas PR, Tizard ML, et al. Two-year-outcomes analysis of Crohn's disease treated with rifabutin and macrolide antibiotics. J Antimicrob Chemother 1997;39:393-400

3 Shafran SD, Singer J, Zarowny DP, et al. Determinants of rifabutinassociated uveitis in patients treated with rifabutin, clarithromycin, and ethambutol for Mycobacterium avium complex bacteremia: a multivariate analysis. Canadian HIV Trials Network Protocol 010 Study Group. J Infect Dis 1998;177:252-5
4 Arevalo JF, Russack V, Freeman WR. New ophthalmic manifestations of presumed rifabutin-related uveitis. Ophthalmic Surg Lasers 1997;28:321-4

5 Bhagat N, Read RW, Rao NA, Smith RE, Chong LP. Rifabutinassociated hypopyon uveitis in human immunodeficiency virus-negative immunocompetent individuals. Ophthalmology 2001;108:750-2

6 Saran BR, Maguire AM, Nichols C, et al. Hypopyon uveitis in patients with acquired immunodeficiency syndrome treated for systemic Mycobacterium avium complex infection with rifabutin. Arch Ophthalmol 1994;112:1159-65

\section{Prosthetic joint infection by cat scratch}

\author{
Jac Ciampolini FRCS ${ }^{1}$ Marina Morgan FRCPath ${ }^{2}$ \\ John Timperley FRCSEd ${ }^{3}$
}

J R Soc Med 2004;97:441-442

In a patient with a joint prosthesis, animal-related injuries present special hazards. Antimicrobial prophylaxis may be needed after a bite or scratch - but with what?

\section{CASE HISTORY}

A woman of 73 was admitted after two days of pyrexia and general malaise, some fourteen months after a routine knee arthroplasty. She also reported a flu-like illness and rigors 12 days previously. She was generally unwell, with a temperature of $37.7^{\circ} \mathrm{C}$ and a painful, red, hot and swollen knee. She had a peripheral blood neutrophilia, and her C-reactive protein was raised at $>110 \mathrm{mg} / \mathrm{L}$. With a provisional diagnosis of septic arthritis the knee joint was aspirated, and microscopy of the fluid revealed $>10^{5}$ polymorphs per $\mathrm{mL}$ but no organisms. Treated empirically with intravenous flucloxacillin, she underwent arthroscopic washout of the knee with $10 \mathrm{~L}$ warmed saline. The next day, despite clinical improvement, the knee remained swollen and painful. Blood cultures were sterile, but after 24 hours Pasteurella multocida was isolated from the joint fluid. She then remembered being scratched by her pet cat on the dorsum of the hand two weeks previously (Figure 1). The antibiotic therapy was changed to intravenous benzyl penicillin $1.8 \mathrm{~g}$ four

'Department of Orthopaedic Surgery, Derriford Hospital, Plymouth; ${ }^{2}$ Department of Microbiology, Royal Devon \& Exeter Hospital, Exeter; ${ }^{3}$ Princess Elizabeth Orthopaedic Hospital, Exeter, UK

Correspondence to: Dr Marina Morgan, Old Pathology Laboratory, Church Lane, Exeter EX2 5AD, UK

E-mail: marina.morgan@rdehc-tr.swest.nhs.uk 